ОШИБКИ: КЛИНИЧЕСКИЕ И МОЛЕКУЛЯРНО-ГЕНЕТИЧЕСКИЕ ХАРАКТЕРИСТИКИ ПАЦИЕНТОВ С НАРУШЕНИЕМ ФОРМИРОВАНИЯ ПОЛА 46,ХУ, ОБУСЛОВЛЕННЫМ МУТАЦИЯМИ В ГЕНЕ NR5A1

[Проблемы эндокринологии. 2020;66(3):62-69. https://doi.org/10.14341/probl12445

( Н.Ю. Калинченко ${ }^{1 *}$, А.А. Колодкина' , Н.Ю. Райгородская², А.Н. Тюльпаков

'Национальный медицинский исследовательский центр эндокринологии, Москва, Россия

${ }^{2}$ Саратовский государственный медицинский университет им. В.И. Разумовского, Саратов, Россия

В статье «Клинические и молекулярно-генетические характеристики пациентов с нарушением формирования пола 46,XY, обусловленным мутациями в гене NR5A1», опубликованной в Т. 66 № 3 журнала «Проблемы Эндокринологии» за 2020 г. (doi: 10.14341/probl12445), допущены ошибки:

- в аннотации «Среди обнаруженных мутаций 15 ранее описаны не были» была исправлена информация на «Среди обнаруженных мутаций 22 ранее описаны не были»;

- в подразделе РЕЗУЛЬТАТЫ «У 36 пациентов было выявлено 31 вариант в гене NR5A1, 15 из которых ранее описаны не были» внесены изменения на «У 36 пациентов было выявлено 31 вариант в гене NR5A1, 22 из которых ранее описаны не были»;

- в подразделе РЕЗУЛЬТАТЫ «Среди впервые выявленных вариантов изменений в гене NR1A1 два приводят к образованию стоп-кодона - p.Y197X и p.Y25X, два к сдвигу рамки считывания - p.N385SfsX10 и p.L245AfsX53, что не позволяет сомневаться в их патогенности. Среди ранее неописанных вариантных изменений 5 миссенсмутаций (р.C283Y, р.C283F, p.H24Q, р.M126K, р.А82T) и 1 синонимичная замена, затрагивающая сайт сплайсинга (Е330Е), были оценены как патогенные, а 5 других - как вероятно патогенные» была исправлена информация на «Среди впервые выявленных вариантов изменений в гене NR1A1 два приводят к образованию стоп-кодона p.Y197X и p.Y25X, два к сдвигу рамки считывания - p.N385SfsX10 и p.L245AfsX53, что не позволяет сомневаться В их патогенности. Среди ранее неописанных вариантных изменений 5 миссенс-мутаций (p.C283Y, p.C283F, p.H24Q, p.M126K, p.A82T ) и 1 синонимичная замена, затрагивающая сайт сплайсинга (Е330Е), были оценены как патогенные, а 5 других — как вероятно патогенные»;

внесены исправления в таблицу 2.

Приведенная ранее информация в статье не должна была сколь-нибудь существенно отразиться на восприятии информации читателями и/или интерпретации представленных данных. Авторы выражают сожаления за неверную информацию в опубликованной ранее статье.

КЛЮЧЕВЫЕ СЛОВА: нарушение формирования пола, гипоспадия, стероидогенный фактор, NR5A1, дисгенезия гонад

\title{
ERRATUM: CLINICAL AND MOLECULAR CHARACTERISTICS OF PATIENTS WITH 46,XY DSD DUE TO NR5A1 GENE MUTATIONS
} (Probl Endokrinol (Mosk). 2020 Sep 16;66(3):62-69. Russian. doi: 10.14341/probl12445)

(c) Natalia Yu. Kalinchenko* , Anna A. Kolodkina' ${ }^{1 *}$ Nadezda Yu. Raygorodskaya ${ }^{2}$, Anatoly N. Tiulpakov ${ }^{1}$

'Endocrinology Reserch Centre, Moscow, Russia

${ }^{2}$ Saratov State Medical University, Saratov, Russia

In the article some corrections were needed.

Abstract: "Heterozygous SF1 variants were found in 36 out of 310 (11.6\%) of cases, among them 15 were not previously described". has been corrected to read "Heterozygous SF1 variants were found in 36 out of 310 (11.6\%) of cases, among them 22 were not previously described".

Results: "Heterozygous SF1 variants were found in 36 out of 310 (11.6\%) of cases, among them 15 were not previously described", has been corrected to read "Heterozygous SF1 variants were found in 36 out of 310 (11.6\%) of cases, among them 22 were not previously described".

Among the newly identified variants in the NR1A1 gene, two lead to the premature stop codon -p. Y197X and p. Y25X, two lead to a shift in the reading frame-p. N385fs and p. L245fs, which does not allow us to doubt their pathogenicityAmong the previously undescribed variant changes, 5 missense mutations (p. C283Y, p. C283B, p.H24Q, p.M126K, p.E81K) and 1 synonymous substitution affecting the splicing site (E330E) were evaluated as pathogenic, and 5 others as probably pathogenic. 
Has been corrected to read: Among the newly identified variants in the NR1A1 gene, two lead to the premature stop codon - p. Y197X and p. Y25X, two lead to a shift in the reading frame - p.N385SfsX10 and p.L245AfsX53, which does not allow us to doubt their pathogenicity

Among the previously undescribed variants, 5 missense mutations (p.C283Y, p.C283F, p.H24Q, p.M126K, p.A82T) and 1 synonymous substitution affecting the splicing site (E330E) were predicted as pathogenic, and 5 others as probably pathogenic by calculating pathogenicity.

The authors apologize for these errors.

KEYWORDS: SF1; steroidogenic factor 1; gonadal dysgenesis; disorders of sex development; hypospadias; NR5A1

Таблица 2. Фенотипические, гормональные и молекулярно-генетические характеристики пациентов.

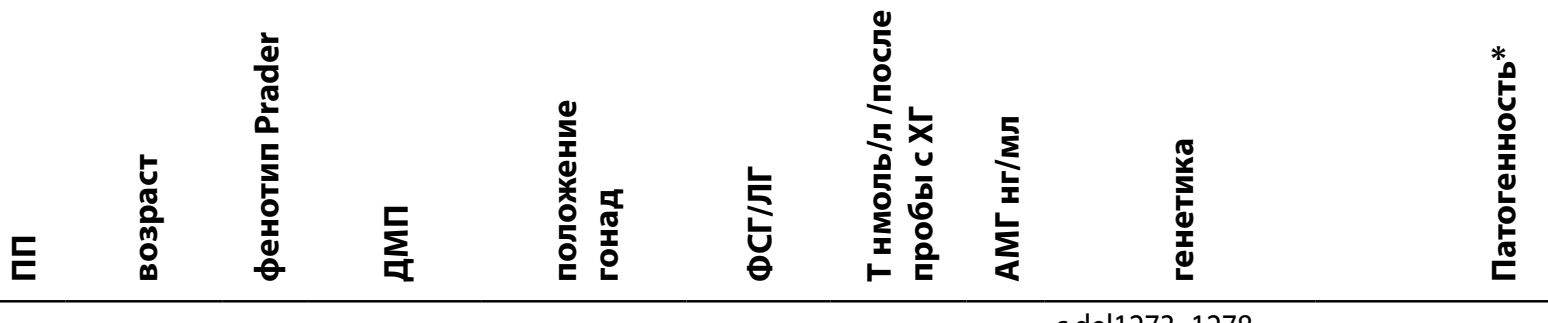

\begin{tabular}{|c|c|c|c|c|c|c|c|c|c|c|}
\hline 1 & ж & $4 \mathrm{mec}$ & III & & & $10.6 / 2.2$ & $1.3 / 7.59$ & 12 & $\begin{array}{l}\text { c.del1273_1278 } \\
\text { p.E425_VI426del }\end{array}$ & П (PS4, PP4, PM2, PVS1) \\
\hline 2 & ж & $11 \mathrm{мес}$ & III & нет & нет & $14 /$ & 10.6 & 13.7 & $\begin{array}{l}\text { c.1152_1153ins(25) } \\
\text { p.N385SfsX10 }\end{array}$ & П (PP4, PM2, PVS1) \\
\hline 3 & ж & 1 год & III & рудимент & ЛГ-МТ, ППК & $6.72 / 0.21$ & 0.36 & 17.6 & c.671C>T:p.P224L & ВП (PS4, РM2, PP2, PP4) \\
\hline 4 & ж & 4 года & ж & есть & & & & & c.104G>A:p.G35D & \\
\hline 5 & ж & 13 лет & II & рудимент & & & & & c.1025C>T:p.S342L & ВП (PS4, PM2, PP2, PP4) \\
\hline 6 & ж & 13 лет & II & & БГ & & & & c.1003A>C:p.T335P & ВП (PS4, РM2, PP2, PP4) \\
\hline 7 & ж & 3 мес & II & нет & ПК & $3.93 / 1.22$ & $1.3 / 13.3$ & 41 & c.848G>T:p.C283F & П (PS4, PM2, PM5, PP2, PP4) \\
\hline 8 & ж & 16 лет & II & нет & ПК & $34 / 27.7$ & 15.7 & & c.937C>T:p.R313C & \\
\hline 9 & ж & 1 г. 4 мес & III & нет & БГ & $0.87 / 0.2$ & 0.1 & 20 & c. $103-3 C>A$ & \\
\hline 10 & ж & 10 мес & $\|$ & нет & ПК & $3.6 / 1.5$ & $0.22 / 24$ & 40 & c.70C>G:p.H24D & $\begin{array}{l}\Pi \text { (PS4, PM2, PM5, PP3, PM1, } \\
\text { PP2, PP4) }\end{array}$ \\
\hline
\end{tabular}

\begin{tabular}{|c|c|c|c|c|c|c|c|c|c|c|}
\hline 11 & ж & 1 г. 5 мес & III & нет & $\begin{array}{c}\text { ППК, } \\
\text { ЛГ- мошонка }\end{array}$ & $10.5 / 2.16$ & $0.5 / 4.51$ & & c.37T>C:p.C13R & \\
\hline 12 & ж & 15 лет & ж & & ПБК, ЛПК & $85 / 43$ & 9.5 & & c.72C>G:p.H24Q & П (PS4, PM2, PM5, PP2, PP4) \\
\hline 13 & ж & 5 лет & III & нет & ПК & & & & c.251G>A p.R84H & \\
\hline 14 & ж & 4 мес & ж & есть & БГ & $7.0 / 0.5$ & $3.17 / 4.89$ & & c. $102+1 G>T$ & \\
\hline 15 & ж & 10 лет & II & нет & ПК & $5.54 / 0.3$ & $/ 7.45$ & 8.45 & c.106T>C:p.F36L & ВП (PS4, PM2, PP2, PP4) \\
\hline 16 & ж & нет инф. & ж & & & & & & c. $245-1 G>T$ & \\
\hline 17 & ж & нет инф. & III & & & & & & c.848G >A:p.C283Y & П (PS4, PM2, PM5, PP2, PP4) \\
\hline 18 & ж & 10 лет & II & нет & & $10.3 / 3.2$ & 10 & & $\begin{array}{l}\text { c.728_733dupGCATC } \\
\text { p.L245AfsX53 }\end{array}$ & П (PP4, PM2, PVS1) \\
\hline 19 & ж>M & 1,5 мес & IV & & ПК & $7.2 / 4.2$ & $3.1 / 10$ & 18 & c.1139-1G>T & \\
\hline 20 & M & $2 \mathrm{mec}$ & IV & & & $5.03 / 1.69$ & $4.0 / 7.8$ & & c.244G>A:p.A82T & П (PS4, PM2, PM5, PP2, PP4) \\
\hline 21 & M & $1 \mathrm{mec}$ & III & нет & в мошонке & $18.6 / 1.0$ & 0.8 & 18.5 & c.721C>T:p R241W & ВП (PS4, PM2, PP2, PP4) \\
\hline 22 & M & 4 г. & IV & & & $1.64 / 0.1$ & 0.09 & & c. 75 C >A:p.Y25X & П (PP4, PM2, PVS1) \\
\hline 23 & M & 5 мес & IV & & $\begin{array}{c}\text { ППК, } \\
\text { ЛГ- мошонка }\end{array}$ & $5.0 / 2.2$ & $5.6 / 33$ & 44 & c.937C>T:p.R313C & \\
\hline 24 & M & $1 \mathrm{mec}$ & III & & ПК & & 4.3 & & c.937C>T:p.R313C & \\
\hline 25 & M & 13 лет & III & нет & $\begin{array}{c}\text { ППК, } \\
\text { ЛГ- мошонка }\end{array}$ & $14.4 / 5.32$ & 15.9 & & c.T377A:p.M126K & П (PS4, PM2, PM5, PP2, PP4) \\
\hline 26 & M & $4 \mathrm{mec}$ & IV & & в мошонке & $7.2 / 4.2$ & 2.63 & & c.C86T:p.T29M & \\
\hline 27 & M & 10 лет & III & нет & ПК & $1.7 / 0.2$ & 0.26 & 31 & c.591C>A:p.Y197X & П (PP4, PM2, PVS1) \\
\hline
\end{tabular}




\begin{tabular}{|c|c|c|c|c|c|c|c|c|c|c|}
\hline & 듣 & 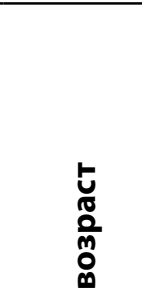 & 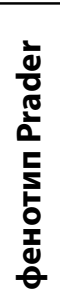 & 듬 & 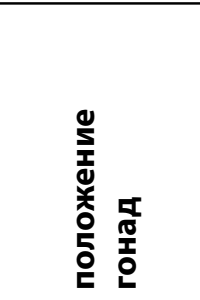 & 는 & 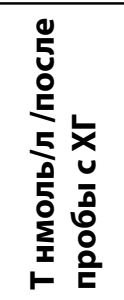 & $\frac{\sum}{\sum}$ & 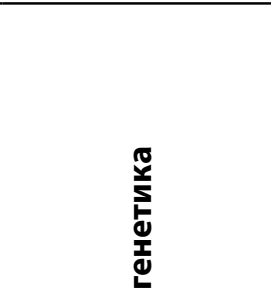 & 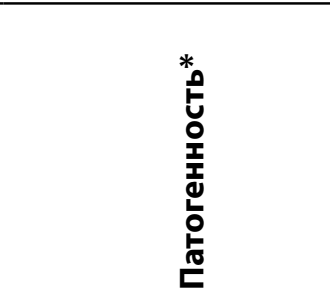 \\
\hline 28 & M & 1 г. 2 мес & III & нет & ПК & $2.4 / 0.48$ & $0.5 / 14$ & & c.C909G:p.S303R & ВП (PM2, РP3, PM1, PP4) \\
\hline 29 & M & 15 лет & III & нет & $\begin{array}{l}\text { ЛПК, ПГ- } \\
\text { в мошонке }\end{array}$ & $9.1 / 8.1$ & 26.2 & 42 & c.G938A:p.R313H & \\
\hline 30 & $M$ & 18 лет & III & нет & ПК & $8 / 5.5$ & 18.3 & 0.6 & c.251G>A:p.R84H & \\
\hline 31 & M & $12 \mathrm{mec}$ & III & нет & & $1.1 / 0.2$ & 0.2 & & c.1289G>A:p.S430N & $\begin{array}{l}\text { ВП (PM2, PM5, PP3, PM1, } \\
\text { PP4) }\end{array}$ \\
\hline 32 & M & 11 лет & III & & & $2.64 / 0.19$ & 0.27 & & c.962G>T:p.G321V & ВП (PM2, РP3, PM1, PP4) \\
\hline 33 & M & 5 мес & IV & & ПК & $2.3 / 0.2$ & $0.03 / 4.6$ & 60.4 & c.251G>A:p.R84H & \\
\hline 34 & M & 18 лет & IV & нет & $\begin{array}{c}\text { ППК, } \\
\text { ЛГ- мошонка }\end{array}$ & $9.6 / 6.9$ & $11 / 20.8$ & & $\begin{array}{l}\text { c.951delC:p. } \\
\text { H317QfsX17 }\end{array}$ & П (PP4, PM2, PVS1) \\
\hline 35 & M & 16 лет & III & нет & ПК & $8 / 5.5$ & 15.4 & & $\begin{array}{l}\text { c.951delC:p. } \\
\text { H317QfsX17 }\end{array}$ & П (PP4, PM2, PVS1) \\
\hline 36 & M & нет инф. & III & & & & & & c.990G>A:p.E330E & П (PM2 PVS1 PP4) \\
\hline
\end{tabular}

Примечания: Т - тестостерон; ЛГ — левая гонада; ПГ — правая гонада; МТ — малый таз; ЛПК — левосторонний паховый крипторхизм; ППК — правосторонний паховый крипторхизм; БГ — большая губа; * - патогенность определена для впервые выявленных вариантных изменений: П патогенная мутация, ВП - вероятно патогенная, в скобках указаны коэффициенты (РР, РМ и др.) для расчета патогенности [12, 13]; ж > м - смена пола с женского на мужской; АМГ >28 нг/мл - норма для мальчиков до 1 года [23].

\section{ИНФОРМАЦИЯ ОБ АВТОРАХ [AUTHORS INFO]}

*Калинченко Наталья Юрьевна, К.M.н. [Natalia Yu. Kalinchenko, MD, PhD,] адрес: Россия, 117036, Mосква, ул. Дм. Ульянова, д. 11 [address: 11 Dm Ulyanova street, 117036 Moscow, Russia]; ORCID: http://orcid.org/0000-0002-2000-7694; eLibrary SPIN: 6727-9653; e-mail: kalinnat@rambler.ru

Колодкина Анна Александровна, K.M.H. [Anna A. Kolodkina, MD, PhD]; ORCID: https://orcid.org/0000-0001-7736-5372; SPIN-код: 6705-6630; e-mail: anna_kolodkina@mail.ru

Райгородская Надежда Юрьевна, д.м.н. [Nadezda Y. Raygorodskaya, MD, PhD];

ORCID: https://orcid.org/0000-0003-0361-5329 SPIN-код: 4227-4358; e-mail: nraygorodskaya@gmail.com

Тюльпаков Анатолий Николаевич, д.м.н. [Anatoly N. Tiulpakov, MD, PhD] Russia];

ORCID: https://orcid.org/0000-0001-8500-4841; SPIN-код: 8396-1798; e-mail: anatolytiulpakov@gmail.com

\section{ЦИТИРОВАТЬ:}

Калинченко Н.Ю., Колодкина А.А., Райгородская Н.Ю., Тюльпаков А.Н Ошибки: Клинические и молекулярногенетические характеристики пациентов с нарушением формирования пола 46,XY, обусловленным мутациями в гене NR5A1. [проблемы эндокринологии 2020;66(3):62-69. doi: 10.14341/probl12445] // Проблемы эндокринологии. 2021. — T. 67. — No6. — C. 124-126. doi: https://doi.org/10.14341/probl12848

\section{TO CITE THIS ARTICLE:}

Kalinchenko NY, Kolodkina AA, Raygorodskaya NY, Tiulpakov AN. Erratum: clinical and molecular characteristics of patients with 46,xy dsd due to nr5a1 gene mutations (Probl Endokrinol (Mosk). 2020 Sep 16;66(3):62-69. Russian. doi: 10.14341/probl12445). Problems of Endocrinology. 2021;67(6):124-126. doi: https://doi.org/10.14341/probl12848 